\title{
Patterning and Identity of Functional Oxide Nanostructures
}

\author{
Zixiao Pan, Vinayak P. Dravid*
}

*Department of Materials Science and Engineering, International Institute of Nanotechnology, Northwestern University, Evanston, Illinois 60208

The ability to create nanoscale architecture for functional oxides is a prerequisite for exploring the rich field of inorganic nanotechnology, given the broad spectrum of their technologically significant properties and their remarkable size dependency [1]. Meanwhile, localized structural characterization and probing of chemical identity as well as the functionality of these nanopatterns are of great significance to understand their properties at miniature scale. Using spinel structured magnetic oxide $\mathrm{CoFe}_{2} \mathrm{O}_{4}(\mathrm{CFO})$ as a model system, we describe here the site-specific fabrication of single crystal CFO nanodisk arrays via "soft electron beam lithography" (Soft- $e$ BL) approach and localized characterization of their crystallinity, chemical composition and magnetic behavior using TEM/STEM and magnetic force microscopy (MFM).

We have recently introduced Soft- $e \mathrm{BL}$ as a novel combination of $e \mathrm{BL}$ and spin coating of liquid precursors for site-specific fabrication of solid-state nanostructures, with high versatility of patterning not only 2-D planar elements but also 3-D hierarchical features $[2,3]$. The SEM and AFM images of the CFO nanodisk arrays patterned on single crystal (100) $\mathrm{SrTiO}_{3}$ substrate via Soft- $e$ BL (annealed at $1000^{\circ} \mathrm{C}$ ) are shown in Fig. 1, indicating the uniform diameter and height of the disks. The magnetic behavior of the CFO patterns was probed using MFM as shown in Fig. 2, in which an area of $5 \times 5$ disk array was scanned before and after magnetization in an out-of-plane static magnetic field. The different phase contrast from the disks before and after magnetization (Fig. 2B-C) indicates the magnetic configuration change influenced by the external field, and thus identifies the active magnetic behavior of the nanopatterns. In order to probe their crystallinity and chemical composition, CFO nanodisks were also patterned on electron transparent $\mathrm{SiN}_{\mathrm{x}}$ membrane for S/TEM investigation. Fig. 3A and B show the STEM images of 40nm-diameter nanopatterns, with the inset diffraction pattern taken from a disk, indicative of their single crystal structure. EDS spectra (Fig. 3C) obtained on the disk and on the plain membrane using nanosized electron probe further confirm the chemical composition of the nanodisks. Thus, the synergistic combination of the versatile Soft- $e$ BL approach and the highly localized identification using fine probes of MFM and S/TEM have successfully demonstrated both, the ability to fabricate nanopatterns and their structural, chemical and functional identity. We believe that such combined approach is imperative to realize the great potential of nanopatterned functional oxides for device-based applications [4].

\section{References}

[1] H. Zheng et al., Science 303 (2004) 661. 
[2] S. Donthu et al., Nano Lett., 5 (2005) 1710.

[3] Z. Pan et al., Small, 2 (2006) 274.

[4] This work was supported by the NSF-NSEC (Award Number EEC-0118025), NSF-MRSEC and the U.S. Department of Energy (DOE-BES). The MFM and S/TEM work were performed in the NIFTI and EPIC facility of the NUANCE Center at Northwestern University. NUANCE Center is supported by NSF-NSEC, NSF-MRSEC, the State of Illinois, and Northwestern University. We also thank Dr. Jian-guo Zheng and Ms. Nasim Alem for help on S/TEM.
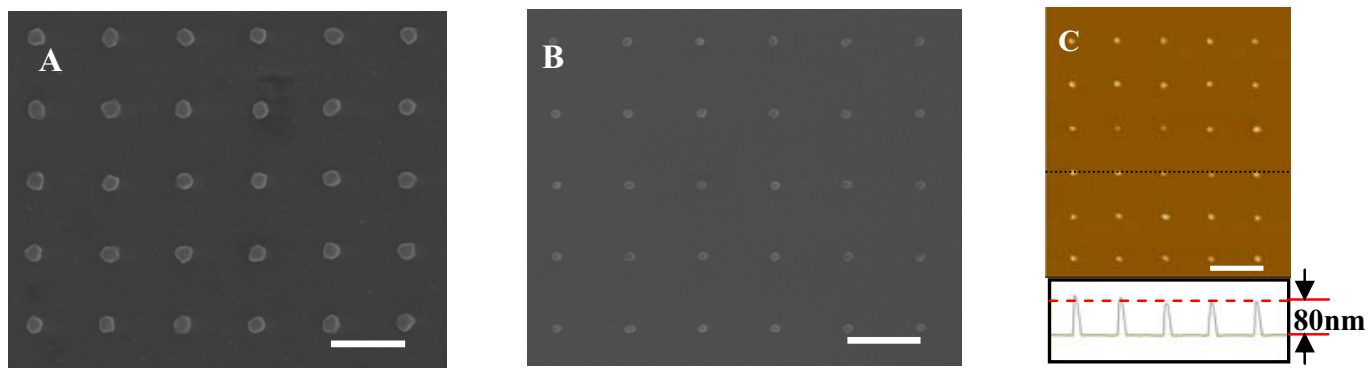

Fig. 1 CFO patterns on single crystal (100) $\mathrm{SrTiO}_{3}$ substrate after $1000^{\circ} \mathrm{C}$ annealing. A. SEM image of $200 \mathrm{~nm}$-diameter patterns. B. SEM image of $100 \mathrm{~nm}$-diameter patterns. C. AFM topography image and cross sectional analysis of $120 \mathrm{~nm}$-diameter patterns. Scale bar $=1 \mu \mathrm{m}$.
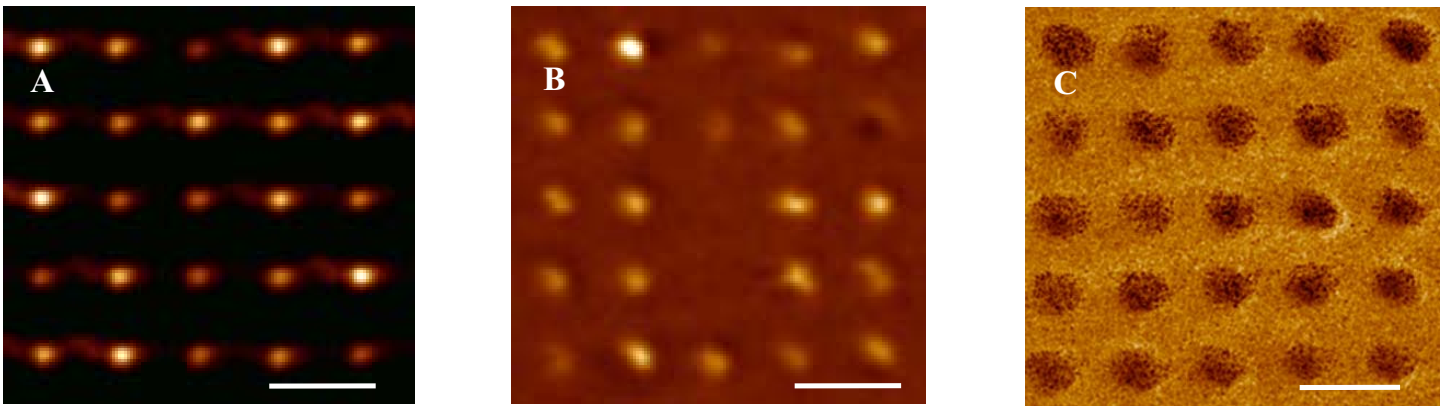

Fig. 2 MFM images of 200nm-diameter CFO patterns on $\mathrm{SrTiO}_{3}$ substrate. A. Topography image. B. Phase image before magnetization. $\mathrm{C}$. Phase image after magnetization under 0.3T static field (field direction pointing into the paper). Scale bar $=1 \mu \mathrm{m}$.
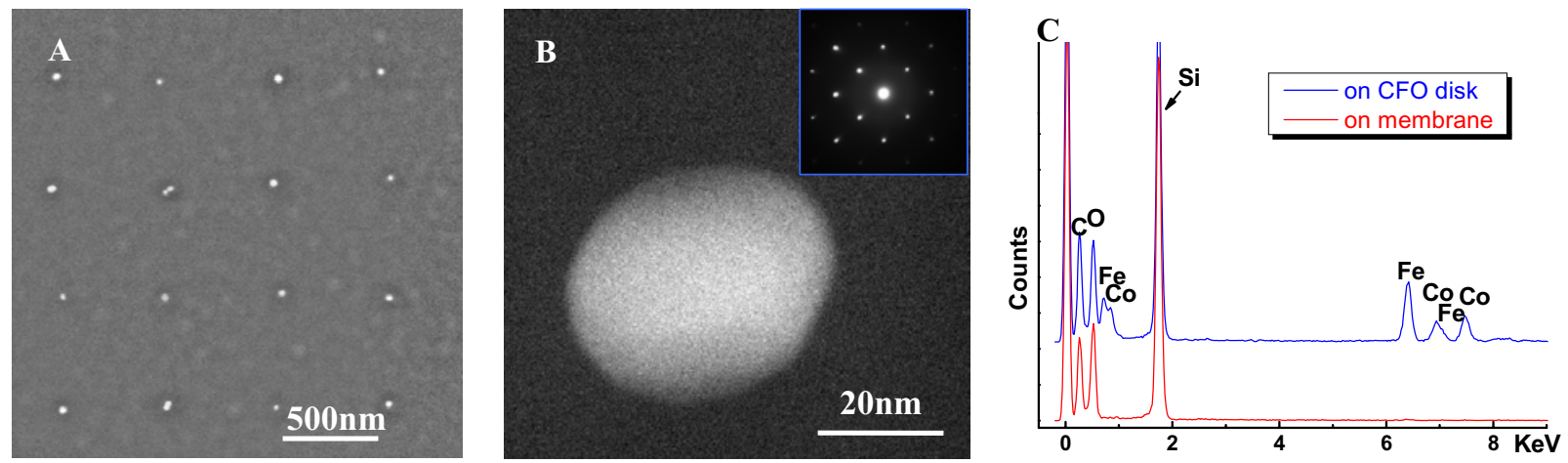

Fig. 3 A. Dark field STEM image of a $4 \times 4$ array of 40nm-diameter CFO disks on SiNx membrane.

B. STEM image of a CFO nanodisk, inset shows the diffraction pattern taken from the nanostructure.

C. EDX spectra taken from the pattern and the plain membrane. 